International Journal of Pure and Applied Mathematics

Volume 97 No. 2 2014, 253-262

ISSN: 1311-8080 (printed version); ISSN: 1314-3395 (on-line version)

url: http://www.ijpam.eu

doi: http://dx.doi.org/10.12732/ijpam.v97i2.14

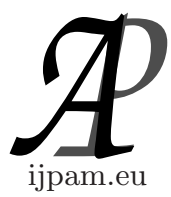

\title{
ON THE $X$-RANK OF A POINTS OF THE TANGENT DEVELOPABLE OF A CURVE IN A PROJECTIVE SPACE
}

\author{
E. Ballico \\ Department of Mathematics \\ University of Trento \\ 38123 Povo (Trento) - Via Sommarive, 14, ITALY
}

\begin{abstract}
Let $X \subset \mathbb{P}^{n}$ be a smooth curve. For any $P \in \mathbb{P}^{n}$ the $X$-rank of $P$ is the minimal cardinality of a set $S \subset X$ such that $P \in\langle S\rangle$, where \langle\rangle denote the linear span. Let $\tau(X) \subset \mathbb{P}^{n}$ be the tangent developable of $X$. We compute upper bounds for the $X$-rank of all $P \in \tau(X)$ or of the general $P \in \tau(X)$, mainly if $X$ is a canonically embedded curve. To do that we define some invariants for the pair $\left(X, \mathcal{O}_{X}(1)\right)$ and compute them if $X$ is canonically embedded and either $X$ is a smooth plane curve or it has general moduli.
\end{abstract}

AMS Subject Classification: 14N05, 14H52

Key Words: tangential developable, $X$-rank, canonical model, smooth plane curve

\section{Introduction}

Fix an integral and non-degenerate variety $X \subset \mathbb{P}^{n}$. For any $P \in \mathbb{P}^{n}$ the $X$-rank $r_{X}(P)$ of $P$ is the minimal cardinality of a subset $S \subset X$ such that $P \in\langle S\rangle$, where \langle\rangle denote the linear span $([16],[8],[15])$. Now assume that $X$ is a smooth curve and that $n \geq 3$. Let $\tau(X) \subset \mathbb{P}^{n}$ be the tangent developable of $X$, i.e. the unions of all tangent lines $T_{O} X$.Quite often the maximal among the

Received: August 6, 2014

(c) 2014 Academic Publications, Ltd. url: www.acadpubl.eu 
integers $r_{X}(P)$ is achieved by a point of $\tau(X) \backslash X$ (e.g. this is the case when $X$ is a rational normal curve by a theorem of Sylvester's ([16, Theorem 4.1], [8, Theorem 23], but it appears quite often elsewhere $([4]))$. In this note we give some criteria to compute upper bounds for points of $\tau(X) \backslash X$ when $X$ is a smooth curve, usually when $X$ is the canonical model of a non-hyperelliptic smooth curve. There are also some easy and well-known lower bound and sometimes we get in this way a good picture). As a byproduct of these definitions we prove the following result.

Corollary 1. Let $C \subset \mathbb{P}^{2}, d \geq 4$, be a smooth pane curve of degree $d$. Let $X \subset \mathbb{P}^{g-1}, g=(d-1)(d-2) / 2$, be the canonical model of $C$ and $\phi: C \rightarrow X$ the canonical map Then $r_{X}(P) \leq 2 d-5$ for all $P \in \tau(X)$ and $r_{X}(P)=d-2$ for a general $P \in \tau(X)$ and $r_{X}(P)=d-3$ for some $P \in \tau(X)$. If $d \geq 5$ and $O \in C$ is such that the tangent line to $C$ at $O$ is neither a flex nor a multitangent, then $r_{X}(P)=d-3$ for at least one and at most $d-2$ points of $T_{\phi(O)}(X) \backslash\{\phi(O)\}$ and $r_{X}(P)=d-2$ for all other points of $T_{\phi(O)}(X) \backslash\{\phi(O)\}$. We have $r_{X}(P) \geq 2 d-6$ for at least one $P \in \tau(X)$.

In section 2 we study the lowest integers $r_{X}(P), P \in \tau(X) \backslash X$. In section 3 we study $r_{X}(P) \in \tau(X)$ (see Proposition 6 for curves with general moduli, Propositions 2, 3 and 5 for an arbitrary curve and Theorem 1 for a result giving, with a little effort, Corollary 1).

We work over an algebraically closed field $\mathbb{K}$ such that $\operatorname{char}(\mathbb{K})=0$.

\section{The Lowest Ranks of Points of $\tau(X) \backslash X$}

Notation 1. Let $C \subset \mathbb{P}^{n}$ be a smooth, connected and non-degenerate curve. Let $\beta(C)$ be the maximal integer such that every zero-dimensional subscheme of $C$ with degree at most $\beta(C)$ is linearly independent.

Let $X$ be a smooth curve of genus $g \geq 3$. The gonality $\operatorname{gon}(X)$ of $X$ is the minimal degree of a line bundle $L$ on $X$ with $h^{0}(X, L) \geq 2$. Let $\operatorname{Gon}(X)$ denote the set of all line bundles $L$ on $X$ with degree $\operatorname{gon}(X)$ and $h^{0}(X, L) \geq 2$. By the definition of gonality we have $\operatorname{Gon}(X) \neq \emptyset$. For each $L \in \operatorname{Gon}(X)$ we have $h^{0}(X, L)=2$ and $X$ is base point free. The set $\operatorname{Gon}(X)$ is a closed subset of $\operatorname{Pic} \operatorname{gon}(X)(X)$ and $\operatorname{dim}(\operatorname{Gon}(X)) \leq 1([11])$. If $X$ is not hyperelliptic and $C \subset \mathbb{P}^{g-1}$ is the canonical model of $X$, then $\beta(C)=\operatorname{gon}(X)-1$.

Fix $L \in \operatorname{Gon}(X)$. Since $L$ is spanned and $h^{0}(X, L)=2$, the complete linear system $|L|$ induces a degree gon $(X)$ morphism $h_{L}: X \rightarrow \mathbb{P}^{1}$. Let $R(L)$ be the set of all $O \in X$ such that $h^{0}(X, L(-2 O))>0$. Since $L$ has no base points, we have 
$h^{0}(X, L(-2 O))=1$ (i.e. $\left.h^{1}(X, L(-2 O))=h^{1}(X, L)+1\right)$ for each $O \in R(L)$ and $h^{0}(X, L(-2 O))=0$ for each $O \in X \backslash R(L)$. For each $O \in R(L)$ let $D_{L, O}$ be the unique effective divisor such that $D_{L, O}+2 O \in|L|$. Since $g>0$ we have $R(L) \neq \emptyset$ for each $L \in \operatorname{gon}(X)$. Let $R(L)^{\prime}$ be the set of all $O \in R(L)$ such that $D_{L, O}$ is reduced (we allow the case in which $O$ appears in $D_{L, O}$, i.e. we allow the case $h^{0}(X, L(-3 O))=1$, but of course $h^{0}(X, L(-4 O))=0$ if $\left.O \in R(L)^{\prime}\right)$. Let $R(L)^{\prime \prime}$ be the set of all $O \in R(L)^{\prime}$ such that $D_{L, O}-O$ is effective; $R(L)^{\prime \prime}$ is the set of all $O \in X$ such that $h^{0}(X(L-3 O))=1$, the unique divisor $D_{L, O}^{\prime}$ of $|L(-3 O)|$ is reduced and $O \notin D_{L, O}^{\prime}$. Set $R(L)_{1}:=R(L)^{\prime} \backslash R(L)^{\prime \prime}$, i.e. let $R(L)_{1}$ be the set of all $O \in X$ with $2 O+S \in|L|$ with $S$ reduced and $O \notin S$. Let $\Delta(X)_{1}$ be the union of all $O \in R(L)_{1}, L \in \operatorname{Gon}(X)$. For each $O \in \Delta_{1}(X)$ let $\mathbb{M}(X, O)$ denote the set of all $L \in \operatorname{Gon}(X)$ such that $O \in R(L)_{1}$. Set $\mu(X, O):=\sharp(\mathbb{M}(X, O))$ with the convention $\mu(X, O)=+\infty$ if $\mathbb{M}(X, O)$ is infinite.

Remark 1. Easy examples (standard cyclic coverings with degree $k$ and large $g$ ) show that we may have $R^{\prime}(L)=\emptyset$ for all $L \in \operatorname{Gon}(X)$. In particular we may have $\Delta(X)_{1}=\emptyset$.

Remark 2. Let $C \subset \mathbb{P}^{n}$ be a smooth curve. Take zero-dimensional schemes $A, B \subset C$. Therefore $A$ and $B$ are effective divisors of $C$. For each $P \in C$ let $a_{P}$ (resp. $b_{P}$ ) be the degree of the connected component of $A$ (resp. $B$ ). The effective divisor $A+B$ has degree $\operatorname{deg}(A)+\operatorname{deg}(B)$ (each $P$ appars with multiplicity $a_{P}+b_{P}$ in $\left.A+B\right)$. The scheme $A \cup B$ is the minimal subscheme of $C$ containing both $A$ and $B$. Each $P \in C$ appears with multiplicity $\max \left\{a_{P}, b_{P}\right\}$ in $A \cup B$. Therefore $A+B=A \cup B$ if and only if $A \cap B=\emptyset$.

Proposition 1. Assume $\operatorname{gon}(X) \geq 6$. We have $r_{X}(P) \geq \operatorname{gon}(X)-2$ for all $P \in \tau(X) \backslash X$. Fix $O \in X$.

(i) If $O \notin \Delta(X)_{1}$, then $r_{X}(P) \geq \operatorname{gon}(X)-1$ for all $P \in T_{O} X \backslash\{O\}$.

(ii) Assume $O \in \Delta(X)_{1}$. Then $\operatorname{gon}(X)-2 \leq r_{X}(P) \leq \operatorname{gon}(X)-1$ for all $P \in T_{O} X \backslash\{O\}$. Let $\mathcal{W}$ be the set of all $P \in T_{O} X \backslash\{O\}$ such that $r_{X}(P)=\operatorname{gon}(X)-2$. We have $\mathcal{W} \neq \emptyset$ and $\sharp(\mathcal{W}) \leq \mu(X, O)$.

Proof. We have $\beta(X)=\operatorname{gon}(X)-1 \geq 5$. Since $\beta(X) \geq 4$, for each $P \in \tau(X)$ there is a unique zero-dimensional scheme $O \in X$ with $P \in T_{O} X$. Fix $P \in \tau(X) \backslash X$ and take the unique $O \in X$ such that $P \in T_{O} X$, i.e. such that $P \in\langle 2 O\rangle$. Fix $S \subset X$ evincing $r_{X}(P)$, i.e. fix $S \subset X$ with $\sharp(S)=$ $r_{X}(P)$ and $P \in\langle S\rangle$. By [7, Lemma 1] we have $h^{1}\left(\mathbb{P}^{g-1}, \mathcal{I}_{2 O \cup S}(1)\right)>0$, i.e. $h^{1}\left(X, \omega_{X}(-(2 O \cup S)) \geq 2\right.$ and hence either $\sharp(S) \geq \operatorname{gon}(X)-1$ or $\sharp(S)=$ gon $(X)-2$ and $O \notin S$ (Remark 2). We get part (i).

Now assume $O \in \Delta(X)_{1}$. Fix $L \in \mathbb{M}(X, O)$ and let $S \subset X$ be the set such 
that $2 O+S \in|L|$. Since $O \notin S$, we have $2 O+S=2 O \cup S$, i.e. $2 O+S$ is the minimal zero-dimensional scheme containing both $2 O$ and $S$. Since $2 O+S$ is a divisor of a line bundle evincing the gonality of $X$, Grassmann's formula gives that $\langle 2 O\rangle \cap\langle S\rangle$ is a single point, $P_{L}$. We get $\mathcal{W} \neq \emptyset$ and the existence of a surjection $\mathbb{M}(X, O) \rightarrow \mathcal{W}$. Hence $\sharp(\mathcal{W}) \leq \mu(X, O)$. Fix any $P^{\prime} \in \mathcal{W}$, say associated to $L \in \Delta(X)_{1}$, and take the only $S \subset X$ such that $2 O+S \in|L|$. We have $O \notin S$ and we saw that $\left\{P^{\prime}\right\}=\langle 2 O\rangle \cap\langle S\rangle$. Fix any $P \in T_{O} X$. Since $P^{\prime} \neq O$, we have $P \in\langle\{O\} \cup S\rangle$ and hence $r_{X}(P) \leq \operatorname{gon}(X)-1$.

\section{The Rank of a General Point of the Tangent Developable}

Let $C$ be a smooth and connected projective curve of genus $g$. We recall the following definitions ([10], [5], [6]). For each integer $r \geq 1$ the $r$-gonality gon $(C, r)$ of $C$ is the minimal integer $d$ such that there is a degree $d$ line bundle $L$ on $C$ with $h^{0}(C, L) \geq r+1$ (note that $h^{0}(C, L)=r+1$ and that $L$ has no base points for each such $L)$. The integer $\operatorname{gon}(C, 1)$ is the gonality $\operatorname{gon}(C)$ of $C$. For every integer $r \geq 2$ the $r$-birational gonality $\operatorname{birgon}(C, r)$ is the minimal integer $d$ such that there is a degree $d$ line bundle $L$ on $C$ with $h^{0}(C, L) \geq r+1$ and the rational map induced by $|L|$ is birational onto its image (notice that $h^{0}(C, L)=r+1$ and $L$ has no base points for each such $\left.L\right)$. For every integer $r \geq 3$ the $r$-embedding gonality embgon $(C, r)$ is the minimal integer $d$ such that there is a degree $d$ line bundle $L$ on $C$ with $h^{0}(C, L) \geq r+1$ and the rational map induced by $|L|$ is an embedding.

If $C$ has general moduli and $1 \leq r \leq g-2$, then $\operatorname{gon}(C, r)=\lceil r g /(r-1)\rceil+$ $r-1$ and the set $W_{d}^{r}(C)$ of line bundles on $C$ evincing $d:=\operatorname{gon}(C, r)$ has pure dimension $\rho(g, r, d):=(r+1) d-r g-r(r+1)$; this set $W_{d}^{r}(C)$ is also irreducible if $\rho(g, r, d)>0([3])$. A dimensional count shows that if $C$ is general and $r \geq 2$, then $\operatorname{gon}(C, r)=\operatorname{birgon}(C, r)$ and that if $\rho(g, r, d)>0$, then a general element of $W_{d}^{r}(C)$ induces a morphism $C \rightarrow \mathbb{P}^{r}$ which is birational onto its image. It is also well-known that if $r \geq 3$, then $\operatorname{gon}(C, r)=\operatorname{embgon}(C, r)$.

Let $C$ be a smooth and connected projective curve of genus $g$. For each $R \in$ $\operatorname{Pic}(C)$ and each $O \in C$ let $d(C, R, O)$ be the minimal integer $d$ such that there is $S \subset C, S$ is reduced, $\sharp(S)=d, h^{0}(C, R(-2 O-S))=h^{0}(R(-2 O))-\sharp(S)+1$ and $h^{0}(C, R(-O-S))=h^{0}(C, R(-O))-\sharp(S)$ (if there is at least one such set $S$; if no set $S$ exists, then we say that $d(C, R, O)$ is not defined). Assume that $d(C, R, O)$ is defined. By Riemann-Roch the last condition is equivalent to $h^{1}(R(-2 O-S))=h^{1}(R(-2 O))+1$. If $R$ is very ample, then $h^{0}(C, R(-2 O))=$ $h^{0}(R)-2$ and hence $h^{1}(C, R)=h^{1}(C, R(-2 O))$. Therefore if $R$ is very ample 
and $D(C, R, O)$ is defined, then $d(C, R, O)$ is the minimal cardinality of a finite set $S$ with $O \notin S, h^{1}(C, R(-2 O-S))>h^{1}(C, R)$ and $h^{1}(C, R(-O-S))=$ $h^{1}(C, R)$. If $d(C, R, O)$ is defined for all $O \in C$ let $d(C, R, 2)$ be the maximum of all integers $d(C, R, O), O \in C$. Let $d^{\prime}(C, R, 2)$ be the minimal integer $d$ such that there is a finite set $A \subset C$ with $d(C, R, O)$ defined and $d \geq d(C, R, O)$ for all $O \in C \backslash A$. Now assume that $C$ has genus $g \geq 3$ and that $C$ is not hyperelliptic., i.e. assume that $\omega_{X}$ is very ample. Set $d(C, O):=d\left(C, \omega_{C}, O\right)$, $d(C, 2):=d\left(C, \omega_{C}, 2\right)$ and $d^{\prime}(C, 2):=d^{\prime}\left(C, \omega_{C}, 2\right)$ (if these integers are defined).

Lemma 1. Let $C$ be a smooth curve and let $R \in \operatorname{Pic}(C)$ be a line bundle with $\operatorname{deg}(R) \geq 4$. Fix $O \in C$ and assume $h^{0}(R(-2 O))=h^{0}(R)-2$, that $R(-2 O)$ is spanned and that the morphism $\phi$ associated to $R(-2 O)$ is birational onto its image. Then $d(C, R, O)$ is defined and $d(C, R, O) \leq h^{0}(R)-1$.

Proof. Since $R(-2 O)$ is spanned, $R$ is spanned at each point of $C \backslash\{O\}$. Since $h^{0}(R(-2 O))=h^{0}(R)-2, R$ is spanned at $O$. Let $\psi: C \rightarrow \mathbb{P}^{r}, r=$ $h^{0}(R)-1$, be the morphism associated to $|R|$. Since $\phi$ is birational onto its image, $\psi$ is birational onto its image. Set $Y:=\psi(C)$ and $B:=\operatorname{Sing}(Y)$. Set $B^{\prime}:=\psi^{-1}(B)$. Notice that $\psi$ induces an isomorphism between $C \backslash B^{\prime}$ and $Y \backslash B$. Since $h^{0}(R(-2 O))=h^{0}(R)-2$, the zero-dimensional scheme $Z:=\psi(2 O)$ has degree two. Hence $\langle Z\rangle$ is a line. Since $\operatorname{deg}(R(-2 O)) \geq 2$, we have $Y \neq\langle Z\rangle$ and hence there is $P \in\langle Z\rangle \backslash\langle Z\rangle \cap Y$. Let $H \subset \mathbb{P}^{r}$ be a general hyperplane containing $P$. Since $P \notin Y$, Bertini's theorem implies that the scheme $Y \cap H$ is formed by $\operatorname{deg}(R)$ distinct points, none of them being in $B \cup\{\phi(O)\}$. Since $P^{\prime} \in\langle Z\rangle \backslash\{\psi(O)\}$, Therefore there is a set $A \subset C \backslash\left(\{O\} \cup B^{\prime}\right)$ with $\psi(A)=Y \cap H$. Since $P^{\prime} \in\langle Z\rangle \backslash\{\psi(O)\}$, we have $\left\langle Y \cap H \cap\langle Z\rangle=\left\{P^{\prime}\right\}\right.$ and $\langle(Y \cap H) \cup\{\psi(O)\})$. Hence $h^{0}(R(-A))=1, h^{0}(R(-O-A))=0$ and $h^{0}(R(-2 O-A))=0$. Therefore $h^{1}(R(-A))=h^{1}(R(-A-O))<h^{1}(R(-A-2 O))$. Hence $d(C, R, O)$ is defined and $d(C, R, O) \leq h^{0}(R)-1$.

Corollary 2. Let $C$ be a smooth curve of genus $g \geq 5$ which is neither hyperelliptic, nor trigonal nor bielliptic. Then $d\left(C, \omega_{C}, 2\right)$ is defined.

Proof. Fix $O \in C$. Since $C$ is not hyperelliptic, the line bundle $\omega_{C}$ is very ample and hence $h^{0}\left(C, \omega_{C}(-2 O)\right)=g-2$. Since $C$ is not trigonal, $\omega_{C}(-2 O)$ is spanned. Let $\phi: C \rightarrow \mathbb{P}^{g-3}$ be the morphism induced by $\left|\omega_{C}(-2 O)\right|$. By Lemma 1 it is sufficient to prove that $\phi$ is birational onto its image. Assume $\operatorname{deg}(\phi) \geq 2$. Since $\phi(C)$ spans $\mathbb{P}^{g-3}$, we have $\operatorname{deg}(\phi(C)) \geq g-3$. Assume for the moment $g \geq 6$. Since $\operatorname{deg}(\phi) \cdot \operatorname{deg}(\phi(C))=2 g-4$ and $g \geq 5$, we get $\operatorname{deg}(\phi)=2$ and $\operatorname{deg}(\phi(C))=g-2$. Since $C$ is not hyperelliptic and $\operatorname{deg}(\phi)=2$, the normalization of $\phi(C)$ is not rational. Since $p_{a}(\phi(C)) \leq 1$ by the upper bound 
for the arithmetic genera of non-degenerate curve in $\mathbb{P}^{r}$ with degree $\leq r+1$, we get that $\phi(C)$ is an elliptic curve. Hence $C$ is a bielliptic curve, a contradiction. Now assume $g=5$. We need to exclude the case $\operatorname{deg}(\phi)=3$. This is excluded, because in this case $\phi(C)$ would be a smooth conic and hence $C$ would be a trigonal curve.

Proposition 2. Let $X \subset \mathbb{P}^{g-1}, g \geq 4$, be a canonically embedded curve such that $d(X, 2)$ is defined. Then $r_{X}(P) \leq d(X, 2)-1$ for all $P \in \tau(X)$.

Proof. It is sufficient to prove the inequality for all $P \in \tau(X) \backslash X$. Fix $O \in X$ such that $P \in\langle 2 O\rangle \backslash\{O\}$. Take a line bundle $L$ on $X$ evincing $d(X, O)$. Therefore we have $2 O+S \in|L|$ with $S$ a reduced divisor and $O$ not in the support of $S$. Hence $\langle 2 O\rangle \cap\langle S\rangle$ is a point, $P^{\prime}$, in $\langle 2 O\rangle \backslash\{O\}$. Since $P \in\left\{P^{\prime}, O\right\}$, we get $r_{X}(P) \leq \sharp(S)-1=d(X, O)-1$.

In the same way we prove the following result.

Proposition 3. Let $X \subset \mathbb{P}^{g-1}, g \geq 4$, be a canonically embedded curve such that $d^{\prime}(X, 2)$ is defined. We have $r_{X}(P) \leq d^{\prime}(X, 2)-1$ for a general $P \in \tau(X)$.

The proof of Proposition 2 and 3 gives the following result.

Proposition 4. Fix a linearly normal smooth curve $C$. If $d\left(C, \mathcal{O}_{C}(1), O\right)$ is defined for all $O \in C$, then $r_{C}(P) \leq d\left(C, \mathcal{O}_{C}(1), 2\right)+1$ for all $P \in \sigma_{2}(C)$. If $d^{\prime}\left(C, \mathcal{O}_{C}(1), 2\right)$ is defined, then $r_{C}(P) \leq d^{\prime}\left(C, \mathcal{O}_{C}(1), 2\right)$ for a general $P \in \tau(C)$.

Proposition 5. We have $d^{\prime}(C, 2) \leq \operatorname{birgon}(C, 2)-3$ for every nonhyperelliptic smooth curve $C$ of genus $g \geq 3$.

Proof. Fix $L \in \operatorname{Pic}(C)$ evincing birgon $(C, 2)$ and let $f: C \rightarrow \mathbb{P}^{2}$ be the associated morphism. Fix a general $O \in C$. Since $O$ is general, $O$ is an ordinary point with respect to $f$ in the sense of [14], i.e. $h^{0}(C, L(-2 O))=1$ and $h^{0}(C, L(-3 O))=0$. Therefore the only divisor $D \in \mid L(-2 O)$ has not $O$ in its support. Since we are in characteristic zero, $f(C)$ is not a strange curve, i.e. there is no $o \in \mathbb{P}^{2}$ contained in every tangent line to a smooth point of $C$. Since $O$ is general, we get $T_{f(O)} f(C) \cap \operatorname{Sing}(C)=\emptyset$. Since a general tangent line of $f(C)$ is tangent at a unique smooth point of $f(C)$ and $f$ is birational onto its image, $D$ contains no point of $C$ with multiplicity $\geq 2$. Since $C$ has positive genus, we have $\operatorname{birgon}(C, 2) \geq 3$. Hence the tangent line of $f(C)$ at $f(O)$ contains another smooth point, say $f(Q)$, of $f(C)$. Take $L^{\prime}:=L(-Q)$ and $S:=D-Q$. We have $h^{0}\left(\mathcal{O}_{C}(2 O)\right)=1$, since $C$ is not hyperelliptic. By construction we have $h^{0}\left(C, \mathcal{O}_{C}(2 O+S)\right)=h^{0}\left(L^{\prime}\right)=2$. 
Theorem 1. Let $C \subset \mathbb{P}^{2}$ be a smooth curve of degree $d \geq 4$. We have $d^{\prime}(C, 2)=d-3$. If the tangent line to $C$ at $O$ is neither a flex nor a multitangent, then $d(C, O)$ is defined and $d(C, O)=d-3$. If the tangent line to $C$ at $O$ is either a flex or a multitangent, then $d(C, O)$ is defined and $d(C, O)=2 d-6$. We have $d(C, 2)=2 d-6$.

Proof. The inequality $d^{\prime}(C, 2) \leq d-3$ follows from Proposition 5 . We have $d^{\prime}(C, 2) \geq d-3$, because $W_{x}^{1}(C)=\emptyset$ for all $x \leq d-2$. The proof of Proposition 5 shows that $d(C, O)=d-3$ if the tangent line to $C$ at $O$ is neither a flex nor a multitangent. Since $W_{d-1}^{1}(C)$ is the set of all $\mathcal{O}_{C}(1)(-O), O \in C$, we get that $d(C, O) \geq d$ if $T_{O} C$ is either a multitangent or an inflexional tangent of $C$. We have $d(C, d) \geq d$, because $C$ has at least one flex. Fix $O \in C$ and assume that the tangent line to $C$ at $O$ is either a flex or a multitangent. Since $\mathcal{O}_{C}(1)(-O)$ has no base points, $\mathcal{O}_{C}(2)(-2 O)$ has no base points. Hence a general $D \in\left|\mathcal{O}_{C}(2)(-2 O)\right|$ is reduced and it does not contain $O$ in its support. Since $h^{i}\left(\mathbb{P}^{2}\left(\mathcal{O}_{\mathbb{P}^{2}}(2-d)\right)=0, i=0,1\right.$, there is a unique conic $T$ with $T \cap C=$ $2 O+D$ (as schemes). Call $Z$ the degree two subscheme of $\mathbb{P}^{2}$ with $2 O$ as its support. A general element of $\left|\mathcal{I}_{Z}(2)\right|$ is a smooth conic. Since $D$ is general, $T$ is a general element of $\left|\mathcal{I}_{Z}(2)\right|$. Therefore $T$ is a smooth conic. We have $h^{0}\left(C, \mathcal{O}_{C}(2)(-2 O-S)\right)=h^{0}\left(C, \mathcal{O}_{C}(2)(-E)\right)=2$, because the smoothness of the conic $T$ implies that $E$ is not formed by 4 collinear points. Therefore $d\left(C, \omega_{C}, O\right)$ is defined and $d(C, O) \leq 2 d-6$. Assume $d(C, O) \leq 2 d-7$, i.e. $d(C, O)+2 \leq 2 d-5$. Take $S$ evincing $d(C, O)$. We have $O \notin S, h^{0}\left(C, \mathcal{O}_{C}(2 O+\right.$ $S))=2$ and $\mathcal{O}_{C}(2 O+S)$ has no base points. Hence $d(C, O)+2$ is in the Lüroth semigroup of $C$. Since $d(C, O)+2 \leq 2 d-5$, S. Greco and G. Raciti proved that $d(C, O)+2 \in\{d-1, d\}([12],[9])$. We excluded the case $d(C, O)+2=d-1$, because the tangent line of $C$ at $O$ is either a flex or a multitangent and hence $O \notin \Delta(C)_{1}$. Now assume $d(C, O)+2=d$ and set $R:=\mathcal{O}_{C}(2 O+S)$. By assumption $R$ is a base point free line bundle of degree $d$. Since $T_{O} C$ is either a flex or a multitangent, $2 O+S \notin\left|\mathcal{O}_{C}(1)\right|$. Therefore $R \neq \mathcal{O}_{C}(1)$. Fix a general $A \in|R|$. Since $\left.h^{0}(R)=2,\left|\omega_{C}\right|=\mid \mathcal{O}_{\mathbb{P}^{2}}(d-3)\right)$, we have $h^{1}\left(\mathbb{P}^{2}, \mathcal{I}_{A}(d-3)\right)>0$. Since $\operatorname{deg}(A) \leq 2(d-3)+1$, there is a line $J \subset \mathbb{P}^{2}$ such that $\operatorname{deg}(A \cap J) \geq d-1$ ([8, Lemma 34]). Since $R$ has no base points, we also have $h^{1}\left(\mathbb{P}^{2}, \mathcal{I}_{A^{\prime}}(d-3)\right)=0$ for every $A^{\prime} \subsetneq A$ (Riemann-Roch and the adjunction formula). Therefore $A \subset J \cap C$. Since $\operatorname{deg}(A)=d$, we get $A \in\left|\mathcal{O}_{C}(1)\right|$ and hence $R \cong \mathcal{O}_{C}(1)$, a contradiction.

Proof of Corollary 1. Fix $O \in C$ such that the tangent line $T_{O} C$ of $C$ at $O$ is neither a tangent line to $C$ ad $O$ is neither a flex of $C$ nor a multitangent of $C$. We have $T_{O} C \cap C=2 O+S$ with $S$ a finite set of $C$ with cardinality $d-2$ and 
$O \notin S$. We have $\mathbb{M}(C, O)=\left\{\mathcal{O}_{C}(1)(-Q)\right\}_{Q \in S}$. Since $C$ has positive genus, the line bundles $\mathcal{O}_{C}(1)(-Q)$ and $\mathcal{O}_{C}(1)\left(-Q^{\prime}\right)$ are not isomorphic if $Q \neq Q^{\prime}$. Therefore $\mu(C, O)=d-2$. Use part (ii) of Proposition to get that $r_{X}(P)=d-3$ for at least one and at most $d-2$ points of $T_{\phi(O)}(X) \backslash\{\phi(O)\}$. Fix $Q \in S$ and set $S^{\prime}:=S \backslash Q$. Since $h^{0}\left(\mathcal{O}_{C}\left(2 O+S^{\prime}\right)\right)=h^{0}\left(C, \mathcal{O}_{C}(1)(-Q)\right)=2$, the set $T_{O} X \cap\left\langle S^{\prime}\right\rangle$ is a single point, $P^{\prime}$, and $P^{\prime} \neq O$. Fix any $P \in\left\langle T_{\phi(O)} X\right.$. Since $P^{\prime} \neq O$, we have $P \in\left\langle\left\{P^{\prime}, \phi(O)\right\}\right\rangle$. Therefore $P \in\langle\{O\} \cup S\rangle$. Hence $r_{X}(P) \leq d-2$. Now take $O$ such that $T_{O} C$ is either a flex or a multitangent of $C$. Theorem 5 gives the existence of $P^{\prime} \in T_{\phi(O)} X \backslash \phi(O)$ with $r_{X}\left(P^{\prime}\right)=2 d-6$. Since $P^{\prime} \neq \phi(O)$ as above we get $r_{X}(P) \leq 2 d-5$ for all $P^{\prime} \in T_{\phi(O)} X \backslash \phi(O)$.

It is often easier to compute the integer $d^{\prime}(C, 2)$ then the integer $d(C, 2)$. It may also be $d(C, 2) \gg d^{\prime}(C, 2)$.

Lemma 2. Let $C$ be a smooth curve of genus $g \geq 2$ for which $d^{\prime}(C, 2)$ is defined. Then $\operatorname{dim}\left(W_{d^{\prime}(C, 2)+2}^{1}(C)\right) \geq 1$.

Proof. If $W_{d^{\prime}(C, 2)+2}^{2}(C) \neq \emptyset$, then $\operatorname{dim}\left(W_{d^{\prime}(C, 2)-+1}^{1}(C)\right) \geq 1$ and hence we have $\operatorname{dim}\left(W_{d^{\prime}(C, 2)+1}^{1}(C)\right) \geq 1$ in this case. Now assume $W_{d^{\prime}(C, 2)+2}^{2}(C)=\emptyset$. In this case we have $G_{d^{\prime}(C, 2)+2}^{1}(C)=W_{d^{\prime}(C, 2)+2}^{1}(C)$. Since any non-constant morphism $f: C \rightarrow \mathbb{P}^{1}$ has only finitely many ramification points, then

$$
\operatorname{dim}\left(G_{d^{\prime}(C, 2)+2}^{1}(C)\right) \geq 1 .
$$

Proposition 6. We have $d^{\prime}(X, 2)=\lfloor g / 2\rfloor$ for a general curve $X$ of genus $g \geq 7$.

Proof. Take an odd integer $g \geq 7$ and set $k:=(g+3) / 2$. We have $\rho(g, 1, k)=2 k-g-2=1, \rho(g, 1, k-1)<0$ and $\rho(g, 2, k)<0$. Therefore Gieseker-Petri and Fulton-Lazarsfeld theorems say that $W_{k}^{1}(X)$ is a smooth and irreducible projective curve and that every $L \in W_{k}^{1}(X)$ is base point free and with $h^{0}(L)=2([3])$. Fix a general $L \in W_{k}^{1}(X)$ and call $f: X \rightarrow \mathbb{P}^{1}$ the associated morphism. For a general $L \in W_{k}^{1}(X)$ the map $f$ has only ordinary ramification and no two ramification points have the same $f$-image. The Riemann-Hurwitz formula gives that $f$ has $2 k+2 g-2=3 g+1$ ramification points. It is sufficient to prove that these ramification points are not the same for a general pair $(L, R) \in W_{k}^{1}(X) \times W_{k}^{1}(X)$. Assume that these ramification points are the same for all general $(L, R)$. Let $\mathcal{M}_{g}^{0}$ the open part of the moduli space $\mathcal{M}_{g}$ of smooth curves formed by the curves without non-trivial automorphisms. Over $\mathcal{M}_{g, 0}$ we would get a degree $3 g+1$ multisection of the morphism $\pi_{1}$ : $\mathcal{M}_{g, 1} \rightarrow \mathcal{M}_{g}$, contradicting the fact that any such rational multisection has a 
degree multiple of $2 g-2$ ([1], [2], [13], [17]) (we have $3 g+1<4 g-4$, because $g \geq 7)$. Since $\rho(g, 1, k-1)<0$, Lemma 2 gives $d^{\prime}(X, 2)(X) \leq k-2$.

Now assume that $g$ is even. We have $\rho(g, 1, g / 2+1)=0$ and hence Lemma 2 gives $d^{\prime}(C, 2) \geq g / 2+2$. We have $\operatorname{dim}\left(W_{g / 2+2}^{1}(X)\right)=\rho(g, r, g / 2+2)=2$. We use the proof of the odd genus case. In this case we have $2(g / 2+2)+2 g-2=3 g+2$ ramification points and we use that $3 g+2<4 g-4$, because we assumed $g \geq 8$.

Question 1. In the set-up of Proposition 6 it is reasonable to conjecture that $d(X, 2)=d^{\prime}(X, 2)$ for a general smooth curve of genus $g \geq 7$.

\section{Acknowledgements}

The author was partially supported by MIUR and GNSAGA of INdAM (Italy).

\section{References}

[1] E. Arbarello, On the Picard group of the moduli space of algebraic curves. Conference on algebraic varieties of small dimension (Turin, 1985), Rend. Sem. Mat. Univ. Politec. Torino 1986, Special Issue, 131-136 (1987).

[2] E. Arbarello and M. Cornalba, The Picard groups of the moduli spaces of curves. Topology 26 (1987), no. 2, 153-171.

[3] E. Arbarello, M. Cornalba, P. Griffiths and J. Harris, Geometry of Algebraic curves, I. Springer, Berlin, 1985.

[4] E. Ballico, On the symmetric tensor rank of non-tangential points of projective curves, Int. J. Pure Appl. Math. 64 (2010), no. 2, 187-189.

[5] E. Ballico, On the gonality sequence of smooth curves, Arch. Math. 99 (2012), 25-31 DOI 10.1007/s00013-012-0409-8

[6] E. Ballico, On the birational gonalities of smooth curves, Annales Universitatis Mariae Curie-Sklodowska Vol. 68 (2014) no. 1, 11-20. doi: 10.2478/umcsmath-2014-0002

[7] E. Ballico and A. Bernardi, Decomposition of homogeneous polynomials with low rank, Math. Z. 271 (2012), 1141-1149; DOI 10.1007/s00209-0110907-6. 
[8] A. Bernardi, A. Gimigliano and M. Idà, Computing symmetric rank for symmetric tensors, J. Symbolic. Comput. 46 (2011), 34-55.

[9] M. Coppens, The existence of base point free linear systems on smooth plane curves, J. Algebraic Geom. 4 (1995), no. 1, 1-15.

[10] H. Lange and G. Martens, On the gonality sequence of an algebraic curve, Manuscripta Math. 137 (2012), 457-473.

[11] Fulton, William; Harris, Joe; Lazarsfeld, Robert Excess linear series on an algebraic curve. Proc. Amer. Math. Soc. 92 (1984), no. 3, 320-322.

[12] S. Greco and G. Raciti, The Lüroth semigroup of plane algebraic curves. Pacific J. Math. 151 (1991), no. 1, 43-56.

[13] A. Kouvidakis, The Picard group of the universal Picard varieties over the moduli space of curves. J. Differential Geom. 34 (1991), no. 3, 839-850.

[14] D. Laksov, Wronskians and Plücker formulas for linear systems on curves, Ann. Scient. Ècole Norm. Sup. (4) 17 (1984), no. 1, 45-66.

[15] J. M. Landsberg, Tensors: Geometry and Applications. Graduate Studies in Mathematics, Vol. 128, Amer. Math. Soc. Providence, 2012.

[16] J. M. Landsberg and Z. Teitler, On the ranks and border ranks of symmetric tensors. Found. Comput. Math. 10 (2010) no. 3, 339-366.

[17] S. Schröer, The strong Franchetta conjecture in arbitrary characteristics, Internat. J. Math. 14 (2003), no. 4, 371-396. 